\title{
Dynamic Analysis of Semi-Circular Dry-Joint Masonry Arches: Small- Scale Experiment and Discrete Element Modeling
}

\author{
TOLGA ATAR ${ }^{1}$, THERESA MCCABE $^{1}$, ECE ERDOGMUS $^{2,}$ AND BORA PULATSU ${ }^{3}$ \\ ${ }^{1}$ Graduate Research Assistant, ${ }^{2}$ Professor, ${ }^{3}$ Postdoctoral Fellow, \\ Durham School of Architectural Engineering and Construction \\ University of Nebraska-Lincoln \\ Scott (Omaha) Campus, Omaha, Nebraska \\ USA
}

\begin{abstract}
This study presents a numerical investigation of the dynamic behavior of dry-joint unreinforced semi-circular masonry arches using the discrete element method (DEM). Masonry arches are analyzed as a system of rigid blocks, mechanically interacting with each other through relative contact displacements. First, the applied modeling strategy is validated using a small-scale tilting experiment performed on a 3D printed arch model and a custom-made rotatable platform. Then, quasi-static and dynamic analysis analyses are performed on computational models to better understand the seismic capacity of the masonry arches depending on the frequency content of the excitations, thickness-to-radius ratios, scale of the arch, and the contact stiffness values. The results of the analyses reveal the capabilities of the discrete element models on the simulation of masonry arches. Particular findings include these types of arches' vulnerability to excitations lower than $2 \mathrm{~Hz}$ dominant frequency. Furthermore, the impact of scale and slenderness become more pronounced for frequencies greater than $2 \mathrm{~Hz}$. Finally, it is observed that once contact stiffness values are larger than 10 $\mathrm{GPa} / \mathrm{m}$, the effect of this parameter is negligible; but it should be carefully selected at lower values.
\end{abstract}

Keywords: Discrete Element Modeling, Masonry Arch, Harmonic Excitations, Dynamic Analysis

Received: November 16, 2019. Revised: May 2, 2020. Accepted: June 18, 2020. Published: July 15, 2020.

\section{Introduction}

Historical masonry arches, whether free-standing or otherwise, are susceptible to failure against the seismic excitations, and understanding possible modes of failure under dynamic loading is crucial for their preservation. Many historic masonry structures have been exposed to a great number of seismic events throughout their lifespan and have survived. Parametric numerical analyses such as those presented here can help understand how they have avoided failure during strong earthquakes and how they can continue to be preserved for the future. Understanding unreinforced masonry arches' response to base excitations will enable a better selection of conservation and rehabilitation decisions to ensure their continued survival.

The discrete element method has been employed since the early $1990 \mathrm{~s}$ as an alternative solution to the continuum-based modeling approaches (i.e., Finite Element Analysis) for the dynamic analysis of masonry structures, including unreinforced masonry arches [1]-[4]. Recently, Dimitri et al. [5] analyzed multi-drum columns and arches-buttress systems subjected to step and harmonic base excitations. They conducted a parametric study to assess failure domain sensitivity as well as the dynamic behavior of a masonry arch on monolithic and multi-block buttresses. Friction properties of joints, geometrical proportions, impulse shape, and the presence of spandrel fill were taken into account. It was found that long period excitations are more threatening than excitations with short periods. The arches subjected to harmonic impulses were more stable than under step impulses, most likely because of the energy dissipation occurring during harmonic excitations. Along a similar vein, Sarhosis et al. [6] used DEM for the analysis of single and multi-drum columns subjected to both horizontal and vertical harmonic earthquake excitations. It was found that multi-drum columns could resist higher levels of base acceleration during high rather than low frequency excitations. The effect of higher friction angles between the drums was analyzed, which resulted in failure by rocking, while a lower friction angle between drums resulted in failure due to shear, which was also observed via quasi-static analysis [7]. Erdogmus et al. [8] simulated an ancient temple subjected to harmonic excitations via discrete element modeling to observe the complex dynamic response of the structure and to predict the possible collapse mechanism of the structure due to various ground motions.

Recently, Erdogmus et al. [9] used DEM to analyze a free-standing ancient Roman masonry 
arch in Usak, Turkey using two models: one representing the deteriorated arch and another portraying its idealized state. This was done to observe how current voussoir imperfections would impact in-plane and out-of-plane failure. Results showed that low frequency excitations triggered smaller acceleration amplitudes both in-plane and out-of-plane and that the undeteriorated geometry performed better than the idealized model, especially in the out-of-plane direction. Three real earthquake records were then used as ground excitations to represent different highly damaging seismic events to predict the effect of different frequencies and amplitudes on the arch's stability.

It is worth noting that the discrete element modeling has an advantage compared to continuumbased modeling strategies since it directly represents the morphology of the masonry arches considering the existing joints. Furthermore, all necessary failure mechanisms that may develop through the dynamic behavior of the structure can be captured using DEM, such as joint opening, sliding, and total loss of contacts, whereas continuum-based models describe the masonry as an equivalent orthotropic continuum associated to either plasticity or damage constitutive law.

In this study, a free-standing semi-circular stone masonry arch is analyzed under harmonic excitations. A parametric analysis is performed to better understand the seismic response of unreinforced semi-circular masonry arches, including the effect of slenderness, scale, and input parameters of excitations. The arch model is first validated using a small-scale 3D printed arch model. This provides a contribution to the field as experimental validation of DEM models is difficult and somewhat rare. The choice of a generic semicircular arch is intentional, as it represents a large body of historical arches from classical, Romanesque, Renaissance and Neo-Classical periods around the world. Note that presented comprehensive analysis of masonry arches provides valuable contributions for the discontinuum type of analyses performed on the dry-joint masonry arches by indicating the influence of essential input parameters on the macro behavior and capacity of the masonry arches.

\section{Computational Model}

In this study, a discontinuum type of analysis is performed considering the masonry arch as a system of rigid discrete blocks, where each block can interact with the adjacent block along their boundaries. For each block representing an individual stone voussoir, dynamic equations of motions (both for translation and rotation) are solved numerically via the central difference algorithm to calculate the central translational and rotational velocities. After integrating the velocities, new positions of the blocks are found. Accordingly, relative contact displacements (along the normal and shear directions) are calculated among the adjacent blocks in order to calculate the contact forces. In the normal direction, a linear behavior in compression (no failure) with null tensile capacity is assumed. The Coulomb-Slip joint model is considered in the shear direction, which requires contact surface friction angle and cohesion. Since the interest of the study is the dry-joint masonry arches, there is no cohesion and tensile strength defined at the contact points. Elastic contact forces are calculated based on the defined contact stiffnesses in the normal and shear directions, and the contact conditions are updated at each step during the analysis. Quasistatic results are obtained from the dynamic equations considering artificial damping by means of dynamic relaxation techniques [10]. In this study, commercial software, 3DEC, developed by ITASCA, is used to perform the numerical analyses [11]. Further information about the theoretical background and applications of DEM, readers are referred to [12]-[15].

The baseline discrete element model used in this study is shown in Fig. 1, consisting of two support blocks and 16 voussoirs. The geomaterial properties of the given circular arch can be defined by determining the radius $(\boldsymbol{R})$, from the center to midthickness of the arch barrel, the thickness of the voussoirs $(\boldsymbol{t})$, and the embrace angle $(\boldsymbol{\alpha})$. In the present study, dynamic analysis is performed via harmonic waves (10 cycles), including different frequency contents (ranging from 0.5 to $6 \mathrm{~Hz}$ ). The dynamic sine waves are applied to the supports as velocity-time histories, and their magnitudes are scaled gradually with $0.01 \mathrm{~g}$ increments until the structure reaches to collapse mechanism, similar to the process followed in Erdogmus et al. [9]. In the dynamic analysis, zero damping is used [16] [17]. 


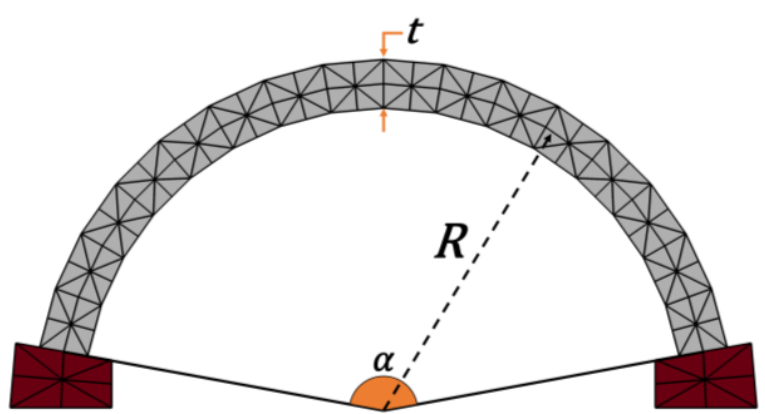

Fig. 1. Masonry Arch - Discrete Element Model

\section{Validation of the Numerical Model: Tilting Test}

To validate the discrete element models and the proposed modeling strategy, small-scale tilting tests are performed using a 3D printed masonry arch model (Fig. 2). A custom-made platform with an embrace angle of 140 degrees is built for this experimental study (Fig. 2).

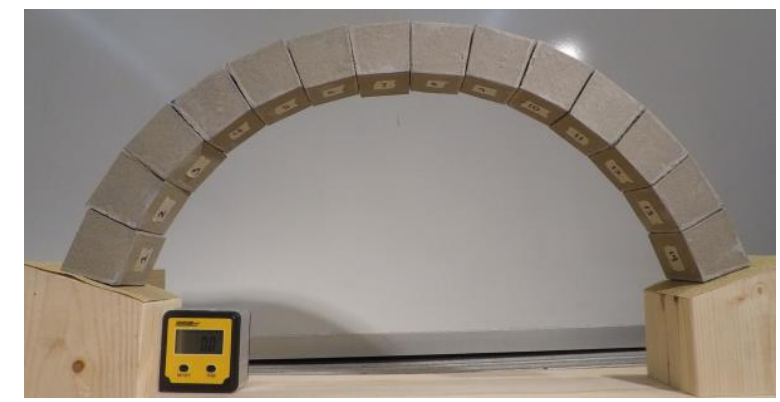

Fig. 2. 3D Printed Arch

A high polymer resin is used to manufacture the 3D printed blocks that are drilled and infilled with fine silica sand to ensure the desired density (around $760 \mathrm{~kg} / \mathrm{m}^{3}$ ). Then, friction spray paint is used to increase the frictional resistance between each block to eliminate excessive sliding failure [18]. The 3D printed semi-circular arch has a 0.43 -meter clear span, 0.15-meter rise, and 0.032-meter arch thickness. During the experiment, the angular deflection (denoted as $\boldsymbol{\beta}$ ), indicating the ratio of the horizontal acceleration to vertical acceleration, of the rotating reference frame is recorded with an electronic measurement device (Fig.3). The tilting test is a quasi-static assessment method, which may be also considered as a first order earthquake assessment, since the examined structures are subjected to constant horizontal force which is calculated as a certain percentage of the weight of the structure based on the tilting angle [19].

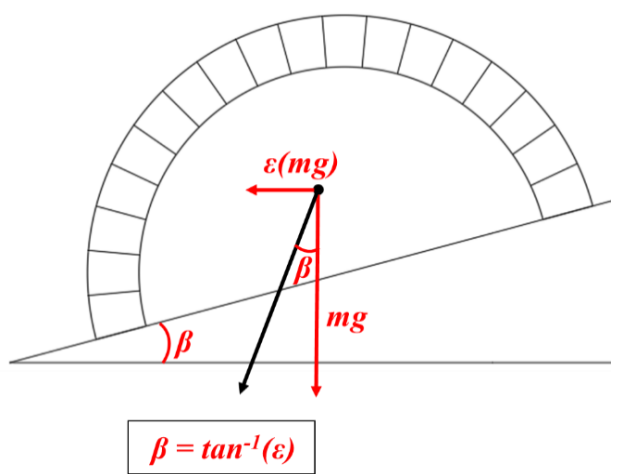

Fig. 3. Schematic representation of the tilting test.

Ten trials are performed for the circular arch model, and the average of the results is compared with the numerical predictions for validation. Similar plastic hinges, either in the extrados or intrados, are observed throughout the experiments (Figures 4 and 5). The final collapse mechanism for the physical model arch is presented in Fig 4. It is important to note it is almost impossible to achieve a perfect arch assemblage due to imperfections at the contact surfaces and slight variations in the block sizes. Moreover, it is quite likely that manual assemblage of the arch models may also lead to some defects on the constructed geometry. Thus, minor changes in the maximum angular deflection and the hinge locations are noted during the ten different trials as expected.

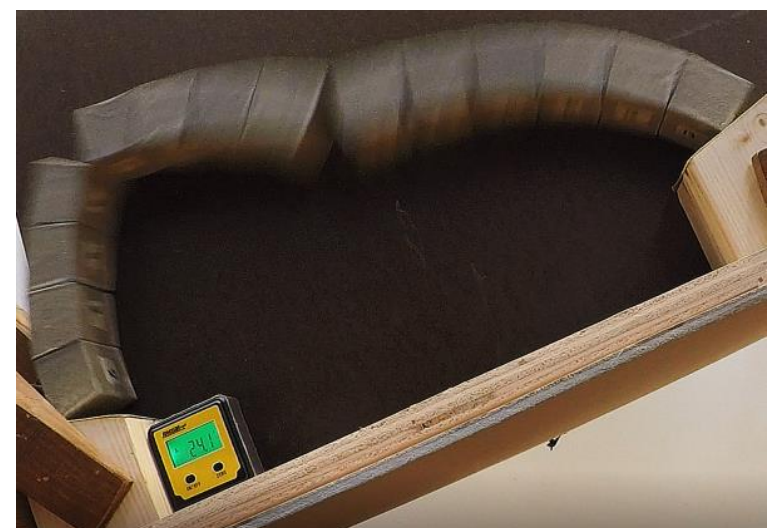

Fig. 4. The collapse mechanism of a small-scale circular arch (24.1 degrees).

In order to take these imperfections into account, a $20 \%$ reduction in the arch thickness is used in the discrete element model, as suggested by DeJong et al. [20], while keeping an identical number of voussoirs. Additionally, the contact stiffness (in the normal and shear directions) is defined as $10 \mathrm{GPa}$ / $m$ to prevent any unphysical penetration among the blocks during the analysis. The final collapse mechanism, obtained from the numerical model 
(Fig. 5), presents a similar behavior as the physical model, shown in Fig. 4, with almost identical hinging locations. Further, the tilting angles obtained from the numerical and physical models differ only by $5.4 \%$ with 25.4 and 24.1 degrees, respectively.

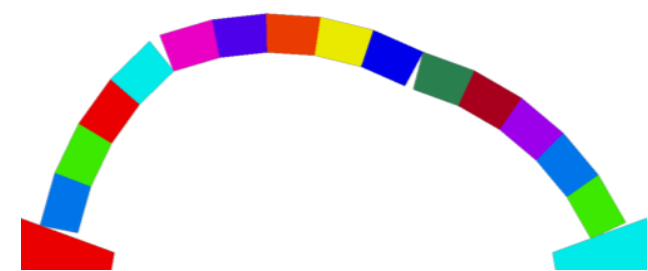

Fig. 5. Collapse mechanism - Discrete element model (25.4 degrees).

According to the results of this validation experiment, it is concluded that the discontinuum analysis of masonry arches utilized in this study provides realistic collapse mechanisms and accurate predictions. Thus, the validated modeling technique is used to investigate the dynamic behavior of this type of masonry arches in the parametric study presented in the next section.

\section{Dynamic Behavior of Masonry Arches under Harmonic Excitations}

Masonry arches exhibit complex dynamic behavior, and the response of the system is directly related to the input excitation parameters. To study these variations in the arch behavior based on the excitation inputs, a parametric analysis is carried out.

First, a discrete arch model $(\boldsymbol{R}=1$ meter, $\boldsymbol{t}=0.15$ meter and $\boldsymbol{\alpha}=160^{\circ}$ ), denoted as the reference arch, is analyzed under unilateral harmonic excitations and quasi-static loading conditions. Contact stiffness is assumed as $50 \mathrm{GPa} / \mathrm{m}$. The results of this analysis are given in Fig. 6, where the vulnerability of the modeled arch to low-frequency seismic actions (i.e., dominant frequency smaller than $2 \mathrm{~Hz}$ ) can be observed. On the other hand, it is obvious that for higher frequency content harmonic waves (i.e., dominant frequency higher than $4 \mathrm{~Hz}$ ), the masonry arch exhibits a better performance in terms of capacity. In other words, peak ground acceleration required to collapse the structure gets higher when the frequency content of the excitation is higher. Furthermore, it is worth noting that the quasi-static solutions are in line with the harmonic excitations within the range of $0.5-2 \mathrm{~Hz}$. It means that pushover (also referred to as nonlinear static analysis) type of analysis of unreinforced dry-joint masonry arches results in similar behavior and capacity compared to dynamic analysis, which has a low dominant frequency content.

A typical collapse mechanism obtained from the dynamic analyses for low frequency harmonic excitation $(0.5 \mathrm{~Hz})$ is shown in Fig. 7. For higher frequency excitations, such as $2.5 \mathrm{~Hz}$ (Fig. 8), rather than a pure hinging mechanism, a mixed collapse mechanism is observed, where sliding failures between the masonry units are observed along with hinging. Therefore, it is indicated that damage and collapse mechanisms of masonry arches may change considerably depending on the characteristics of the excitation.

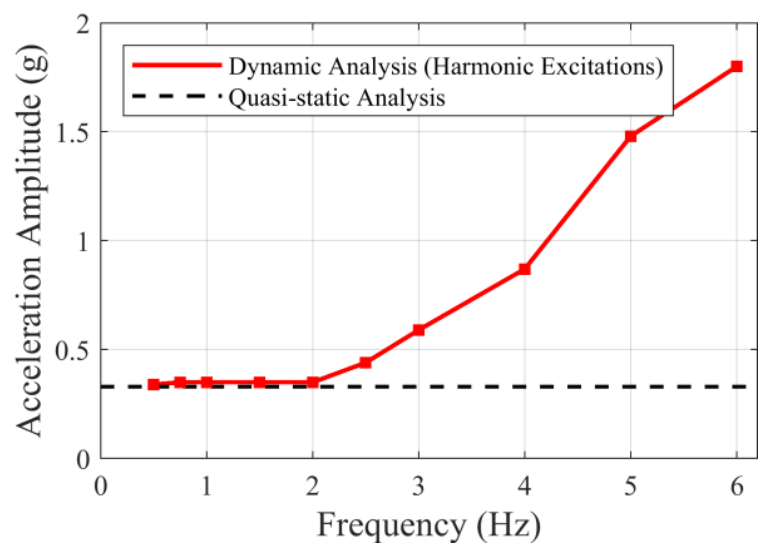

Fig. 6. Safe-unsafe boundary obtained for reference arch.

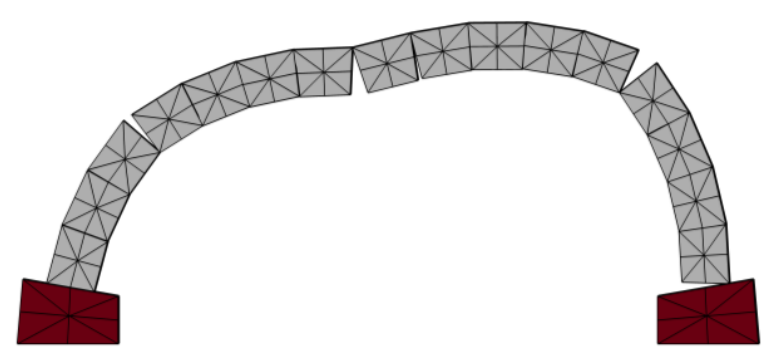

Fig. 7. Hinging mechanism (Dynamic Analysis - Freq. $0.5 \mathrm{~Hz}$ ).

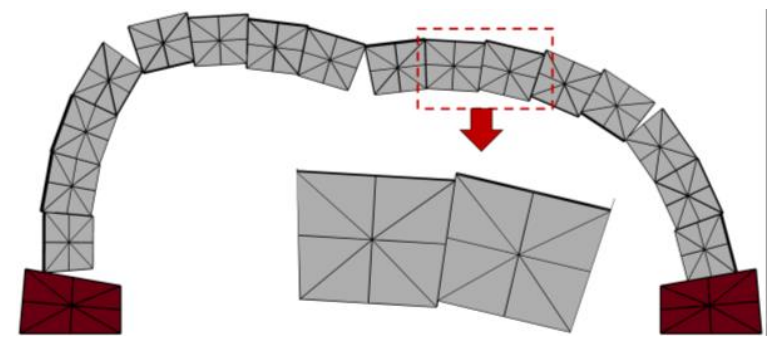

Fig. 8. Sliding + Hinging mechanism (Dynamic analysis - Freq. $2.5 \mathrm{~Hz}$ )

From the material properties point of view, contact stiffness has an important role in the response of discrete element models, especially in the elastic regime, since the elastic forces are 
calculated based on the contact stiffness assigned to each contact point between the adjacent blocks. In order to show the effect of the contact stiffness on the capacity of the structures, a parametric analysis is done, where the contact stiffness is varied from 1 $\mathrm{GPa} / \mathrm{m}$ to $500 \mathrm{GPa} / \mathrm{m}$, as $1,10,25,50,100$ and 500 $\mathrm{GPa} / \mathrm{m}$. A harmonic excitation is then applied with a moderate frequency content $(1.5 \mathrm{~Hz})$. The results (Fig. 9) show that, unless the contact stiffness is smaller than $10 \mathrm{GPa} / \mathrm{m}$, the difference between the results is within $\pm 10 \%$ of each other. Hence, obtained results do not exhibit a significant difference within the contact stiffness range of 10 $500 \mathrm{GPa} / \mathrm{m}$.

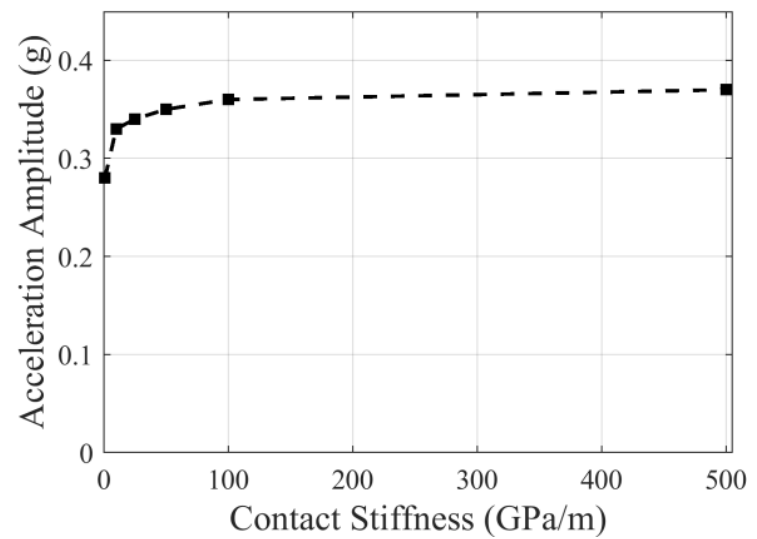

Fig. 9. Influence of contact stiffness on the capacity (Harmonic excitation $-1.5 \mathrm{~Hz}$ )

Finally, the influence of the slenderness and scale of a semi-circular arch is investigated via sensitivity analyses. In Fig. 10, it can be observed that the thicker masonry arches present higher capacity, as expected. Furthermore, the scale effect is investigated by increasing the size of the masonry arch while keeping the same slenderness ratio. The results show that the pronounced influence of the larger-scale models when the frequency content of the excitation gets higher, as shown in Fig. 11.

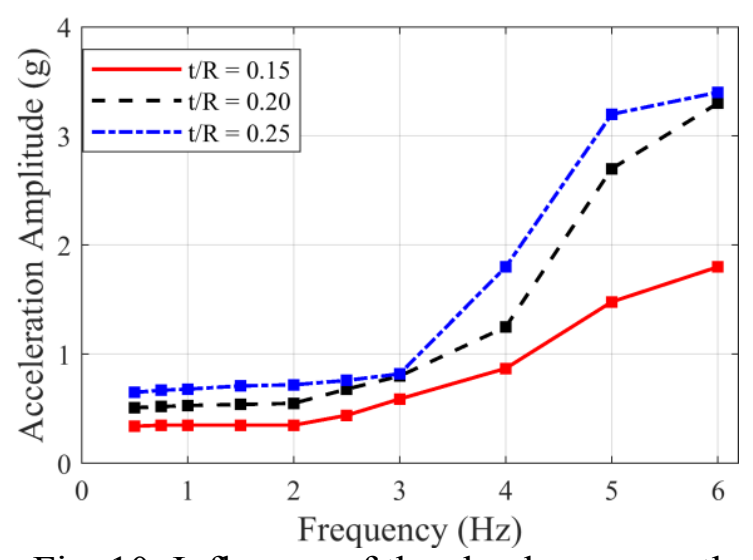

Fig. 10. Influence of the slenderness on the dynamic response of the masonry arches.

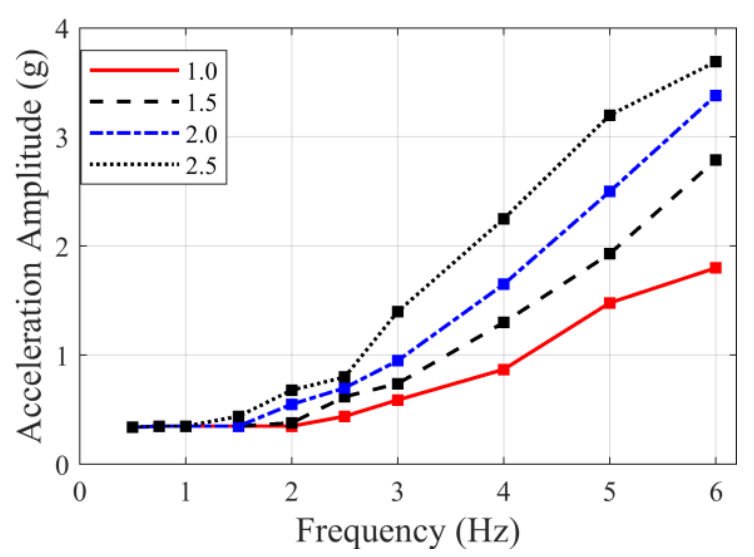

Fig. 11. Scale effect on the dynamic response of the masonry arches.

\section{Conclusions}

This research demonstrates the capability of discrete element modeling in the prediction of the quasistatic and dynamic response of dry-joint masonry arches in terms of capacity and collapse mechanism. First, the small-scale experimental study (quasistatic), namely the tilting test of a $3 \mathrm{D}$ printed arch, is utilized to validate the applied modeling strategy with as small as a $5.4 \%$ difference in tilting angles. Then, the dynamic response of a semi-circular dryjoint masonry arch is explored through a parametric analysis, where excitation frequency, contact stiffness, slenderness (thickness to radius ratio), and scale were varied. The results show the vulnerability of unreinforced semi-circular and dry-joint masonry arches under relatively low frequency (under $2 \mathrm{~Hz}$ ) excitations. Additionally, it is revealed that the quasi-static analyses provide more conservative predictions of the seismic capacity of the masonry arches compared to higher frequency excitations applied as harmonic waves. It was also observed that, with contact stiffness values larger than 10 $\mathrm{GPa} / \mathrm{m}$, the difference between the results is within 
$\pm 10 \%$ of each other, and can be considered negligible. The study shows improved capacity with increased arch thickness. In terms of scale effects, the difference becomes more pronounced under exciting frequencies larger than $1 \mathrm{~Hz}$, but negligible under 1Hz. Future studies of this research will include the arch-pier systems with and without mortar joints. Furthermore, the varying number of voussoirs to explore the threshold for failure under dynamic excitations for arches is another possible direction for future research.

\section{References:}

[1] J. V. Lemos, "Discrete element modeling of the seismic behavior of stone masonry arches," in Computer methods in structural masonry, 1997, no. September, pp. 220-227.

[2] B. Pulatsu, E. Erdogmus, E. M. Bretas, and P. B. Lourenço, "In-Plane Static Response of Dry-Joint Masonry Arch-Pier Structures," in AEI 2019, 2019, pp. 240-248.

[3] B. Pulatsu, E. Erdogmus, and E. M. Bretas, "Parametric Study on Masonry Arches Using 2D Discrete-Element Modeling," J. Archit. Eng., vol. 24, no. 2, p. 04018005, Jun. 2018.

[4] H. Alexakis and N. Makris, "Hinging Mechanisms of Masonry Single-Nave Barrel Vaults Subjected to Lateral and Gravity Loads," J. Struct. Eng., vol. 143, no. 6, p. 04017026, 2017.

[5] R. Dimitri, L. De Lorenzis, and G. Zavarise, "Numerical study on the dynamic behavior of masonry columns and arches on buttresses with the discrete element method," Eng. Struct., vol. 33, no. 12, pp. 3172-3188, 2011.

[6] V. Sarhosis, D. Baraldi, J. V. Lemos, and G. Milani, "Dynamic behaviour of ancient freestanding multi-drum and monolithic columns subjected to horizontal and vertical excitations," Soil Dyn. Earthq. Eng., vol. 120, no. October 2018, pp. 39-57, 2019.

[7] B. Pulatsu, V. Sarhosis, E. M. Bretas, N. Nikitas, and P. B. Lourenço, "Nonlinear static behaviour of ancient free-standing stone columns," Proc. Inst. Civ. Eng. Struct. Build., vol. 170, no. 6, pp. 406-418, 2017.

[8] E. Erdogmus, B. Pulatsu, A. Gaggioli, and M. Hoff, "Reverse Engineering a Fully Collapsed Ancient Roman Temple through Geoarchaeology and DEM," Int. J. Archit. Herit., vol. 00, no. 00, pp. 1-21, Feb. 2020.

[9] E. Erdogmus, B. Pulatsu, B. Can, and K. Ozkan, "Analysis of the Last Standing Arch of the Roman Aqueduct at Blaundos," in 13th North American Masonry Conference, 2019, no. June, pp. 483-493.

[10] J. V. Lemos, "Block modelling of rock masses. Concepts and application to dam foundations," Rev. Eur. Génie Civ., vol. 12, no. 7-8, pp. 915-949, Oct. 2008.

[11] Itasca Consulting Group Inc., "3DEC Three Dimensional Distinct Element Code." Minneapolis, 2013.

[12] R. Hart, P. A. Cundall, and J. V. Lemos, "Formulation of a three-dimensional distinct element model-Part I. Mechanical calculations for motion and interaction of a system composed of many polyhedral blocks," Int. J. Rock Mech. Min. Sci., vol. 25, no. 3, pp. 107-116, Jun. 1988.

[13] P. A. Cundall, "Formulation of a threedimensional distinct element model-Part I. A scheme to detect and represent contacts in a system composed of many polyhedral blocks," Int. J. Rock Mech. Min. Sci. Geomech., vol. 25, no. 3, pp. 107-116, 1988.

[14] J. V. Lemos, "Discrete element modeling of masonry structures," Int. J. Archit. Herit., vol. 1, no. 2, pp. 190-213, 2007.

[15] B. Pulatsu, E. M. Bretas, and P. B. Lourenço, "Discrete element modeling of masonry structures: Validation and application," Earthquakes Struct., vol. 11, no. 4, pp. 563582, Oct. 2016.

[16] E. Tavafi, A. Mohebkhah, and V. Sarhosis, "Seismic Behavior of the Cube of Zoroaster Tower Using the Discrete Element Method," Int. J. Archit. Herit., vol. 0, no. 0, pp. 1-16, 2019.

[17] I. Psycharis, D. Y. Papastamatiou, and A. P. Alexandris, "Parametric investigation of the stability of classical columns under harmonic and earthquake excitations," Earthq. Eng. Struct. Dyn., vol. 29, no. 8, pp. 1093-1109, Aug. 2000.

[18] B. Pulatsu, "Simulation of complex 3D behavior of masonry arch systems (Doctoral dissertation)," University of NebraskaLincoln, 2019.

[19] P. Block, T. Ciblac, and J. Ochsendorf, "Real-time limit analysis of vaulted masonry buildings," Comput. Struct., vol. 84, no. 2930, pp. 1841-1852, 2006.

[20] M. J. DeJong, L. De Lorenzis, S. Adams, and J. A. Ochsendorf, "Rocking Stability of Masonry Arches in Seismic Regions," Earthq. Spectra, vol. 24, no. 4, pp. 847-865, Nov. 2008.

\section{Creative Commons Attribution License 4.0 (Attribution 4.0 International, CC BY 4.0)}

This article is published under the terms of the Creative Commons Attribution License 4.0

https://creativecommons.org/licenses/by/4.0/deed.en_US 